\title{
Riqueza y diversidad de aves en un paisaje Agropecuario en el ejido Chalahuiyapa, Huejutla, Hidalgo.
}

\author{
Richness and diversity birds in a landscape Agricultural in the Ejido Chalahuiyapa, Huejutla, \\ Hidalgo. \\ Cipriano-Anastasio Juan ${ }^{1 凶}{ }^{\bowtie}$, López-Mancilla Alejandra ${ }^{1}$, Cabrera-Martínez Dorismilda ${ }^{1}$ \\ y Capistrán-Barradas Ascención ${ }^{2}$ \\ ${ }^{1}$ Licenciatura en Biología, Instituto Tecnológico de Huejutla. Carretera Huejutla- \\ Chalahuiyapa Km 5.5, CP. 43000, Huejutla de Reyes, Hidalgo, México. \\ ${ }^{2}$ Facultad de Ciencias Biológicas y Agropecuarias, Universidad Veracruzana, Carretera \\ Tuxpan Tampico Km 7.5, Col. Universitaria, CP. 92850 Tuxpan, Veracruz, México \\ ${ }^{凶}$ Autor para correspondencia: bio.jca@gmail.com
}

Recibido: $18 / 02 / 2017$

Aceptado: 30/06/2017

\section{RESUMEN}

La pérdida de los hábitats afecta seriamente a la diversidad biológica. Se realizó un estudio de riqueza y diversidad de aves en un paisaje agropecuario, durante los meses que comprenden a la estación húmeda, (otoño-invierno). Usando trayectos lineales se registraron 50 especies de aves, 10 órdenes y 22 familias. El mes más rico en especies y diverso fue octubre con 22.02 especies efectivas y el hábitat con mayor abundancia, riqueza y diversidad fueron la vegetación secundaria con 24.1 y acahual 14.4 especies efectivas. Los estimadores Jack 1 y Jack 2 revelan que para la zona la mayor riqueza es 69 (72\%) y $80(62 \%)$ especies. Estos estimadores dan una equivalencia total de $67 \%$ de la avifauna total de los cuatro hábitats muestreados. La semejanza entre vegetación secundaria y acahual nos revelan que comparten un $36 \%$ de las especies y que no se encuentran en hábitats perturbados, maizal y pastizal.

Palabra Clave: Riqueza, diversidad, especies efectivas, hábitat.

\begin{abstract}
The loss of habitats seriously affects biodiversity. A study of the richness and diversity of birds was carried out in an agricultural landscape during the months that comprise the wet season (autumnwinter). Using linear trajectories, 50 species of birds, 10 orders and 22 families were registered. The most rich and diverse month was October with 22.02 effective species and the habitat with greater abundance, richness and diversity were the secondary vegetation with 24.1 and finally 14.4 effective species. The estimators Jack 1 and Jack 2 reveal that for the zone the greatest wealth is $69(72 \%)$ and $80(62 \%)$ species. These estimators give a total equivalence of $67 \%$ of the total avifauna of the four sampled habitats. The similarity between secondary vegetation and acahual reveals that they share $36 \%$ of the species and are not found in disturbed habitats, corn and pasture.
\end{abstract}


Keywords: Richnnes, diversity, effective species, habitat.

\section{INTRODUCCIÓN}

México es considerado como uno de los países con una notable megadiversidad, ocupa el tercer sitio al nivel mundial y esto es debido a su situación geográfica compleja, su gran heterogeneidad fisiográfica, climática y ecológica (López y Cervantes, 2002). Nuestro territorio nacional alberga una gran riqueza de aves silvestres, con cerca de 1,079 especies, distribuidas en 22 órdenes, 77 familias y 397 géneros (Howell y Web, 1995). Esto hace que sean un componente importante de nuestros paisajes, debido a que estas contribuyen en la dispersión de semillas, polinización de plantas, control de plagas, belleza escénica y como bioindicadoras de la salud de ecosistemas (IMAE, 2006).

La fragmentación siempre está asociada a los efectos negativos derivados de las acciones antrópicas que conllevan a una modificación intensa del territorio y que se traduce en una pérdida importante de hábitats naturales (Martínez, 2009). Esto ha tenido un impacto negativo sobre las poblaciones de aves, reduciendo la diversidad de especies,

interrumpiendo procesos ecológicos y modificando su composición (Cárdenas et al. 2003). Algunos hábitats albergan una cantidad importante de la avifauna original; es evidente que algunos usos del suelo creados y manejados por los productores cumplen un papel importante en el mantenimiento de la comunidad de aves (Sáenz et al. 2007; Enríquez-Lenis et al. 2006). El objetivo del presente estudio radica en cuantificar la riqueza y diversidad espacio-temporal de las aves en cuatro agroecosistemas en el ejido Chalahuiyapa, determinar si estos ecosistemas modificados son prioritarios para su manejo y conservación.

\section{MATERIAL Y MÉTODOS}

El Ejido Chalahuiyapa se localiza en el Municipio, Hidalgo, México, a $120 \mathrm{msnm}$. Abarcando cuatro tipos de hábitats (vegetación secundaria, acahual, pastizal y maizal), se estableció un trayecto lineal de 1 $\mathrm{km} / \mathrm{h}$ por tipo de vegetación (Ralph, 1996). Los recorridos se realizaron entre 7:00 y 10:00 am, que es el periodo con mayor actividad en las aves. Se registró la hora y fecha del censo.

Riqueza y diversidad: La riqueza de especies se basa en el número total de especies obtenido por un censo de la comunidad (Moreno, 2001). Se tomó la medida de diversidad verdadera de orden uno (q1) en la cual todas las especies son consideradas en el valor de diversidad, ponderadas proporcionalmente según su abundancia en la comunidad (Moreno et al. 2011), por medio de la obtención exponencial del índice de entropía de Shannon: ${ }^{1} \mathrm{D}=\exp$ (H') (Jost, 2006). Para calcular la riqueza de especies máxima se utilizaron los estimadores Jack1 y Jack2 los cuales funcionan mediante los datos de presencia-ausencia. Estos solo toman en cuenta las abundancias (Moreno, 2001). Para el Análisis de similitud: Se empleó el coeficiente de similitud de Jaccard para determinar el grado en que dos muestras son semejantes por las especies presentes en ellas. Donde el rango de este índice va desde cero (0) cuando no hay especies compartidas, hasta uno (1) cuando los dos sitios comparten las mismas especies. Este índice mide diferencias en la presencia o ausencia de especies.

\section{RESULTADOS}

Durante los meses que comprenden a la estación húmeda, se registraron 357 individuos, 
repartidos en 10 órdenes, 22 familias y 50 especies. Las familias con más especies fueron: Tyrannidae (6 spp.), Accipitridae (5 spp.), Columbidae y Thraupidae (4 spp.). El orden Paseriformes presentó 30 especies, las especies más abundantes fueron: Dives dives, Icterus gularis y Psilorhinus morio.

Riqueza y diversidad mensual: el mes de septiembre obtuvo una riqueza de 25 especies las más representativas fueron: Columbina inca, Dives dives y Saltator atriceps. Octubre obtuvo 29 especies (Polioptila caerulea, Icteria virens y Cardellina pusilla). Noviembre obtuvo 26 spp; y las más representativas fueron: Psilorhinus morio y Myiozetetes similis, diciembre obtuvo $20 \mathrm{spp} ; y$ enero $19 \mathrm{spp}$. El mes con mayor abundancia fue septiembre, el más rico en especies y en efectivas fue octubre, esto es aunado a la llegada de las aves migratorias a esta zona. Los meses con menor abundancia $y$ especies efectivas fueron: diciembre y enero (Cuadro 1).

Cuadro 1. Riqueza y diversidad mensual de aves en el Ejido Chalahuiyapa.

\begin{tabular}{|c|c|c|c|c|}
\hline Meses & Abundancia & Riqueza & $\begin{array}{c}\text { Shannon } \\
\left(\mathbf{H}^{\prime}\right)\end{array}$ & $\begin{array}{c}\text { Especies } \\
\text { efectivas }\end{array}$ \\
\hline Septiembre & 96 & 25 & 2.818 & 16.74 \\
\hline Octubre & 72 & 29 & 3.092 & 22.02 \\
\hline Noviembre & 87 & 25 & 2.706 & 14.97 \\
\hline Diciembre & 46 & 20 & 2.71 & 15.03 \\
\hline Enero & 56 & 19 & 2.763 & 15.85 \\
\hline
\end{tabular}

Riqueza y diversidad por hábitat: el hábitat vegetación secundaria, obtuvo una riqueza mayor a los demás sitios con 43 especies y 24.1 spp efectivas, las más representativas fueron: Cardellina pusilla, Icterus gularis y Leptotila verreauxi. El hábitat acahual obtuvo una riqueza de 21 especies con $14.4 \mathrm{spp}$; efectivas. Los hábitats pastizal y maizal obtuvieron una menor riqueza $\mathrm{y}$ menor número de $\mathrm{spp}$; efectivas (Cuadro 2).
Cuadro 2. Riqueza y diversidad de aves por hábitat.

\begin{tabular}{|l|c|c|c|c|}
\hline Hábitat & $\begin{array}{c}\text { Abundanci } \\
\mathbf{a}\end{array}$ & $\begin{array}{c}\text { Riquez } \\
\mathbf{a}\end{array}$ & $\begin{array}{c}\text { Shanno } \\
\mathbf{n}\left(\mathbf{H}^{\prime}\right)\end{array}$ & $\begin{array}{c}\text { Especies } \\
\text { efectivas }\end{array}$ \\
\hline $\begin{array}{l}\text { Vegetació } \\
\mathbf{n} \\
\text { secundaria }\end{array}$ & 221 & 43 & 3.18 & 24.1 \\
\hline Acahual & 97 & 21 & 2.66 & 14.4 \\
\hline Pastizal & 24 & 11 & 2.22 & 9.2 \\
\hline Maizal & 15 & 8 & 1.93 & 6.4 \\
\hline
\end{tabular}

Tomando datos de presencia ausencia, en septiembre registró un total de 25 especies con las muestras tomadas y los estimadores Jack 1 y Jack 2 octubre obtuvo $36 \mathrm{spp}$. Por su parte noviembre registró $44 \mathrm{spp}$; los estimadores marcan 57 y $61 \mathrm{spp}$; en diciembre se estiman 65 y 74 de 48 especies obtenidas y finalmente en enero debieron haberse obtenido 69 y 80 spp. (Fig. 1). Estos estimadores reportan una equivalencia total de $67 \%$ de la avifauna total de los cuatro hábitats muestreados.

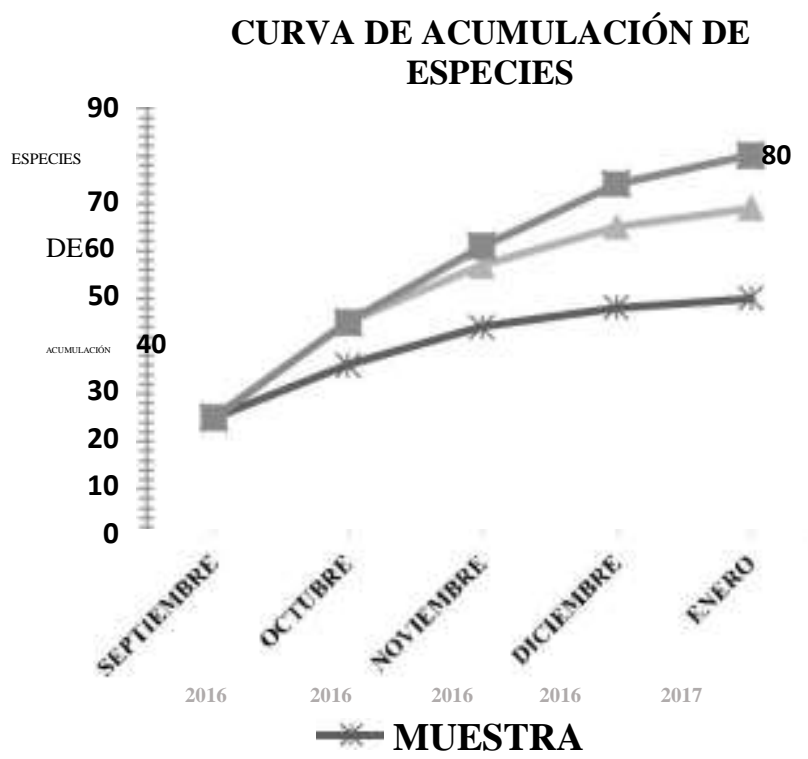

Figura 1. Curva de acumulación de especies de aves en los cuatro tipos de hábitats durante los meses de temporada húmeda en el Ejido Chalahuiyapa. 
El índice de similitud de Jaccard separó el hábitat pastizal de los otros hábitats. El hábitat vegetación secundaria (Veg. Sec.) es similar con el acahual, razón por el cual forman un grupo con un valor de similitud cercano a 0.4 , esto indica que en cuanto a avifauna comparten el 36\% de especies registradas (Fig. 2).
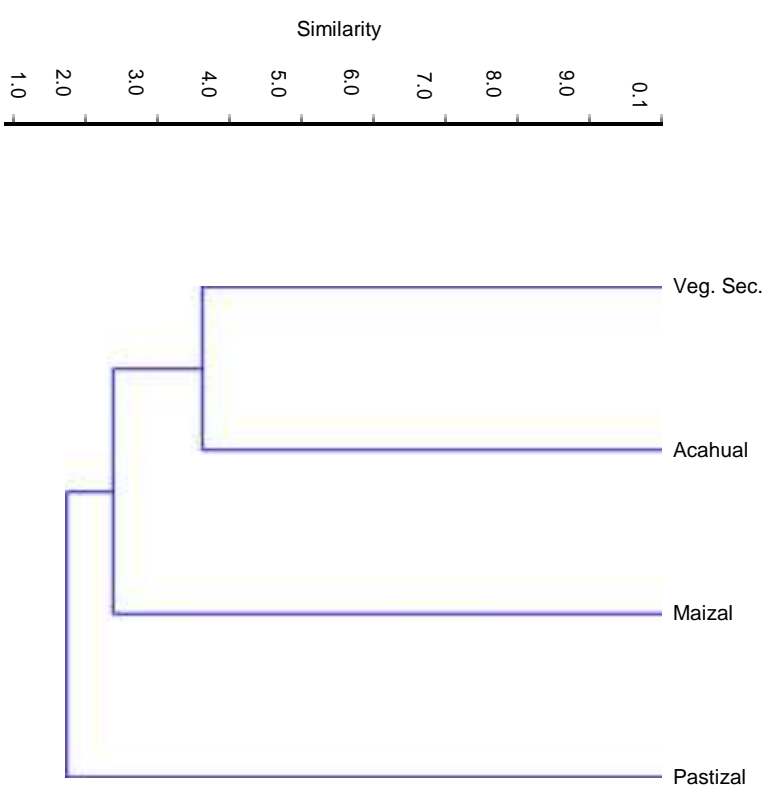

Figura 2. Análisis de similitud de Jaccard de las especies de aves entre los cuatro hábitats.

Las especies que comparten este grupo son: Amazilia yucatanensis, Campylorhynchus zonatus, Cardenilla pusilla, Chrotophaga sulcirostris, Cyanocorax yncas, Dives dives, Icterus gularis, Leptotila verreauxi, Megarrynchus pitangua, Melanerpes aurifrons, Pheugopedius maculipectus, Polioptila caerulea, Psilorhinus morio, Quiscalus

mexicanus, Saltator atriceps, Saltator coerulescens y Sayornis phoebe. Mientras que el maizal y el pastizal comparten pocas especies de hábitats abiertos: Dives dives, Sayornis phoebe y Volatinia jacarina.

Las especies arbustivas y arbóreas más representativas del sistema agropecuario, vegetación secundaria y acahual, se muestran en la siguiente tabla (Cuadro 3).

Cuadro 3. Especies arbustivas y arbóreas representativas de la zona

\begin{tabular}{|c|c|c|}
\hline Especies & $\begin{array}{c}\text { Vegetación } \\
\text { secundaria }\end{array}$ & Acahual \\
\hline Acacia cornigera & & $\mathrm{x}$ \\
\hline Bursera simaruba & $\mathrm{x}$ & \\
\hline Citrus sinensis & & $\mathrm{x}$ \\
\hline Cedrela odorata & $\mathrm{x}$ & $\mathrm{x}$ \\
\hline Carica papaya & & $\mathrm{x}$ \\
\hline $\begin{array}{c}\text { Enterolobium } \\
\text { cyclocarpum }\end{array}$ & $\mathrm{x}$ & \\
\hline $\begin{array}{c}\text { Gliricidia sepium } \\
\text { Guazuma ulmifolia }\end{array}$ & $\mathrm{x}$ & \\
\hline Psidium guajava & $\mathrm{x}$ & $\mathrm{x}$ \\
\hline $\begin{array}{c}\text { Pithecellobium } \\
\text { dulce }\end{array}$ & $\mathrm{x}$ & \\
\hline $\begin{array}{c}\text { Malvaviscus } \\
\text { arboreus }\end{array}$ & $\mathrm{x}$ & $\mathrm{x}$ \\
\hline $\begin{array}{c}\text { Muntingia } \\
\text { calabura }\end{array}$ & $\mathrm{x}$ & \\
\hline
\end{tabular}

\section{DISCUSIÓN}

Al igual que otros estudios realizados en agropaisajes (Cárdenas et al. 2003; EnriquezLennis et al. 2006), la familia Tyrannidae obtuvo mayor número de especies. La abundancia en los diferentes usos del hábitat muestra una dominancia de Dives dives, Myiozetetes similis y Psilorhinus morio en hábitats donde se han realizado modificaciones como en acahual y vegetación secundaria, por otra parte este último sitio presenta buena cobertura arbórea, esto permite una mayor conectividad con los remanentes de selva y a su vez ayuda al mantenimiento y conservación de aves y disminuye los efectos negativos de la fragmentación del hábitat (Cárdenas et al. 2003; Lang et al. 2003). 
Los números efectivos permitieron una mejor interpretación de la diversidad de las comunidades y las comparaciones entre sitios, incorporando datos de abundancia y estos a su vez cumplen con las propiedades acordes con la interpretación intuitiva del concepto biológico de diversidad (Moreno, 2011; Jost 2006). El índice de entropía de Shannon no tiene una explicación biológica clara, es por eso que anteriormente algunas de las razones decidían analizar la diversidad de las comunidades donde consideraban únicamente la riqueza de especies. Lo anterior descrito, nos indica que la riqueza y diversidad de especies está aunada a la gran variedad de árboles presentes (Bursera simaruba, Cedrela odorata, Gliricidia sepium, Guazuma ulmifolia, Psidium guajava y Pithecellobium dulce). Sobre todo en el hábitat vegetación secundaria, esto indica que a mayor riqueza de especies arbórea mayor es el número de aves particulares de selva, debido a que aumenta la variedad de recursos para la avifauna o sitios de protección (Mills et al. 1991). No se registraron especies de aves específicas de selva en maizales o potreros. En esta investigación la baja similitud entre las áreas de estudio, sobre todo en los hábitats maizal y pastizal, sugiere que cada una presenta su propia composición dependiendo el grado de perturbación. Sin embargo la variación de la riqueza son atribuibles al intercambio de especies (Bojorges-Baños y López-Mata, 2006).

Aun cuando la conservación de aves terrestres depende de la compresión de sus requerimientos del hábitat y de los procesos físicos y bióticos que lo mantienen (Askins, 2000), se ha establecido que la combinación de hábitats simples y complejos conducen a un nuevo aprovechamiento de los recursos (Wilson, 1974) y que la diversidad de especies de aves está vinculada a la diversidad del paisaje, por lo que la conservación de este último asegura la conservación y diversidad de especies (Böhning-Gaese, 1997). Por otra parte es importante recalcar que en este estudio los sitios con poca cobertura arbórea como el maizal y pastizal ganadero no aportaron mucho a la riqueza y diversidad de aves. Sin embargo, los sistemas silvopastoriles son una alternativa de manejo sostenible, debido a que poseen importante potencial para proveer hábitats $\mathrm{y}$ recursos e incrementar la conectividad del paisaje agropecuario. Además, pueden permitir el movimiento animal a través de áreas agropecuarias y al mismo tiempo ayudar a incrementar la productividad y diversificación de productos en las zonas ganaderas (De la Ossa, 2013).

\section{CONCLUSIONES Y RECOMENDACIONES}

A pesar de ser un paisaje muy fragmentado, el Ejido Chalahuiyapa cuentan con 50 especies de aves registradas, 22 familias y 10 órdenes. Los hábitats que mantienen una avifauna más diversa son la vegetación secundaria y acahual. El mes abundante fue septiembre, el más rico y diverso fue octubre y el hábitat con mayor abundancia, riqueza y diversidad de aves fue la vegetación secundaria y el acahual. La curva de acumulación de especies nos dice que el esfuerzo de muestreo fue muy bajo y los indicadores Jack 1 y Jack 2 revelan que aún faltan especies por registrar. La similitud entre la vegetación secundaria y el acahual nos revelan que tienen en común el $36 \%$ de avifauna y comparten especies que no se encuentran en los demás hábitats. Se recomienda ampliar el número de horas de muestreo, repeticiones y los meses, abarcando dos temporadas, época de secas y lluvias, esto para tener una mejor representatividad de los datos.

\section{LITERATURA CITADA}

Askins, R. A. 2000. Restoring North America's birds. Yale University Press. 336 p.

Bojorges B, J C. y López-Mata, L. 2006. Asociación de la riqueza y diversidad de especies de aves y estructura de la vegetación en una selva mediana subperennifolia en el centro de Veracruz, México. Revista Mexicana de Biodiversidad 77: 235-249.

https://doi.org/10.22201/ib.20078706e.2006.002 
Böhning-Gaese, K. 1997. Determinants of avian species richness at different spatial scales. Journal of

Biogeography 24:49-60.

https://doi.org/10.1111/j.1365-2699.1997.tb000

Cárdenas, G., Harvey, C.A., Ibrahim, M. y Finegan B. 2003. Diversidad y riqueza de aves en diferentes hábitats en un paisaje fragmentado en Cañas, Costa Rica, Agroforestería en las Américas, (10): (39-40) 78-85.

De la Ossa, L.A. 2013. Cercas vivas y su importancia ambiental en la conservación de avifauna nativa, Rev. Colombiana Cienc. Anim. 5(1):171-193.

https://doi.org/10.24188/recia.v5.n1.2013.483

Enríquez-Lenis, M. L., Sáenz, J. C. y Ibrahim, M. 2006. Riqueza, abundancia y diversidad de aves y su relación con la cobertura arbórea en un agropaisaje dominado por la ganadería en el trópico subhúmedo de Costa Rica. Agroforestería en las Américas (45):49-57.

Howell, S. N. G. y S. Webb. 1995. A guide to the birds of Mexico and Northern Central America. Oxford University Press. 851p.

IMAE. 2006. Avances del estudio de avifauna del centro cultural y recreativo" El Cedazo". Documento

Interno. Instituto del Medio Ambiente. Gobierno del Estado de Aguascalientes. México.

Jost, L. 2007. Partitioning diversity into independent alpha and beta components. Ecology 88:2427-2439. https://doi.org/10.1890/06-1736.1

Martínez, A.C. 2009. Conectividad ecológica y áreas protegidas. Herramientas y casos prácticos. $86 \mathrm{p}$.

Moreno, C. E., Barragán, F., Pineda, E. y Pavón, N. 2011. Reanálisis de la diversidad alfa: alternativas para interpretar y comparar información sobre comunidades ecológicas.
Revista Mexicana de Biodiversidad 82 (4):1249-1261.

https://doi.org/10.22201/ib.20078706e.

Moreno, C. E. 2001. Métodos para medir la biodiversidad. M\&T-Manuales y Tesis SEA, vol. 1. Zaragoza, 84 p.

Lang, I., Gormley, L.H.L., Harvey, C.A y Sinclair, F.L. 2003. Composición de la comunidad de aves en cercas vivas de Rio Frío, Costa Rica. Agroforestería en las Américas. 10(39-40):86-92.

López, B. R. y J. B. Cervantes. 2002. Unidades del paisaje para el desarrollo sustentable y manejo de los recursos naturales. Revista de información y análisis (20):43-49.

Ralph, C.J., Geupel, G.R., Pyle, P., Martin, T.E., Desante, D.F y Millá, B. 1996. Manual de Métodos de Campo para el Monitoreo de Aves Terrestres. General Technical Reports. PSWgtr- 159. Albany, C.A: Pacific Southwest Research Station, Forest Service U.S. Departament of Agriculture. 46 p. https://doi.org/10.2737/PSW-GTR-159

Renjifo, L. M. 1999. Composition changes in a subandean avifauna after long-term Forest

Fragmentation. Conservation biology 13(5):1124-1139 pp.

https://doi.org/10.1046/j.1523-1739.1999.98311 Sáenz, J. C., Villatoro, F., Ibrahim, M., Fajardo, D. y Pérez, M. 2007. Relación entre las comunidades de aves y la vegetación en agropaisajes dominados por la ganadería en Costa Rica, Nicaragua y Colombia. Agroforestería en las Américas (45):37-48.

Willson, M. F. 1974. Avian community organization and habitat structure. Ecology 55:1107-1029.

https://doi.org/10.2307/1940352 
Copyright (c) 2017 Juan Cipriano Anastasio, Alejandra López Mancilla,

Dorismilda Cabrera Martinez y Ascención Capistrán Barradas

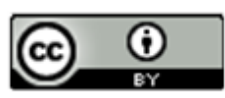

Este tex to está protegido por una licencia licencia Creative Commons 4.0.

Usted es libre para Compartir — copiar y redistribuir el material en cualquier medio o form ato- y Adaptar el documento —remezdar, transformar y crear a partir del material- para cualquier propósito, incluso para fi nes comerciales, siempre que cumpla la condición de:

Atribución: Usted debe dar crédito a la obra original de manera adecuada, proporcionar un enlace a la licencia, e in dicar si se han realizado cam bios. Puede hacerlo en cualquier form a razonable, pero no de form a tal que sugiera que tiene el apoyo del licenciante o lo recibe por el uso que hace de la obra.

Resumendelicencia - Textocompletodelalicencia 